\title{
Thermal conductivity of amorphous polymers and its dependence on molecular weight
}

\author{
Andy Kiesslinga ${ }^{\mathrm{a}}$, David Nieto Simavilla ${ }^{\mathrm{b}}$, Georgios G. Vogiatzis ${ }^{\mathrm{c}}$, David C. \\ Venerus $^{\mathrm{a}, *}$ \\ ${ }^{a}$ Otto H. York Department of Chemical and Materials Engineering, New Jersey Institute of \\ Technology, Newark, NJ 07103, United States \\ ${ }^{b}$ Basque Center for Applied Mathematics, 48009 Bilbao, Universidad de Burgos, 09006 \\ Burgos, Spain \\ ${ }^{c}$ School of Chemical Engineering, National Technical University of Athens, Athens \\ GR-15780, Greece
}

\begin{abstract}
Thermal conductivity is an important transport property governing the performance of polymers in non-isothermal conditions. Nevertheless, its dependence on molecular weight $M$ has not been the subject of as much attention as other properties of polymeric materials. We determine the thermal conductivity of polystyrene and polyisobutylene for a wide range of molecular weight by measuring the density, heat capacity and thermal diffusivity. Using coarse-graining and reverse mapping methods, we were able to produce molecular melts to study the thermal conductivity of polystyrene using molecular dynamics simulations over a similar range of molecular weight. We find satisfactory agreement between the experimental and simulation results. However, all of our results show that thermal conductivity depends only slightly on molecular weight up the entanglement limit and it is independent thereafter. Our results put into question the few previous experimental studies on this topic by showing that the previously accepted proportionality to $\sqrt{M}$ does not hold. Our findings could have significant implications for the understanding of complex phenomena such as anisotropic thermal conductivity in polymers subjected to flow.
\end{abstract}

Keywords: thermal conductivity, molecular weight, polystyrene, polyisobutylene, forced Rayleigh scattering, MD simulation 2010 MSC: 00-01, 99-00

\section{Introduction}

Thermal transport plays a critical role in the processing of polymer melts and in the performance of fabricated polymeric materials. This is a result of most polymer manufacturing processes being non-isothermal in nature. The

*venerus@njit.edu 
5 transport property governing thermal transport is the thermal conductivity $\lambda$, which appears in the expression known as Fourier's law:

$$
\boldsymbol{j}_{q}=-\lambda \nabla T
$$

In Eq. (1), $\boldsymbol{j}_{q}$ is the diffusive flux of energy, and $\boldsymbol{\nabla} T$ is the gradient of temperature $T$. Polymer melts show smaller thermal conductivity than common organic liquids and water, and the difference is even larger for polymeric solids in contrast to ceramic and metallic materials. Here, we use the word 'polymer' to refer to a molecule having at least 10 monomers: $N=M / M_{0}>10$, where $M$ is the polymer molecular weight and $M_{0}$ is the monomer molecular weight (oligomers are molecules with $N \sim 3-10$ ). For typical polymers, values of the thermal conductivity $\lambda$ are in the range $0.1-0.4 \mathrm{~W} / \mathrm{mK}[1]$.

15 Thermal conductivity is a notoriously difficult quantity to measure and this especially true for polymeric liquids and solids. According to Eq. (1), the most direct approach to measuring thermal conductivity $\lambda$ is to subject a sample to a known heat flux and measure the resulting temperature gradient, or vice-versa. The most common methods for measuring the thermal conductivity of polymers include the transient hot wire and guarded hot plate techniques. Literature values for the thermal conductivity of a given polymer (liquid or solid) can vary by $100 \%$ or more [2]. The inconsistencies of thermal conductivity data for polymeric materials may be the result of several factors such as finite geometries (edge effects) and uncontrolled interfacial thermal resistances.

The dependence of thermodynamic and transport properties of polymers on molecular weight has technological and scientific implications. There have been numerous investigations, both experimental and theoretical, on the molecular weight dependence of polymer viscosity $\eta$ and diffusion coefficient $D$. These transport coefficients are related to configurational rearrangements involving an entire polymer chain and therefore have a very strong dependence on molecular weight $M$. For entangled polymers with $M>M_{\mathrm{e}}$, where $M_{\mathrm{e}}$ is the entanglement molecular weight, $\eta \sim M^{3.4}$ and $D \sim M^{-2.3}$, which are somewhat stronger than the predictions of so-called reptation models [3]. The glass transition temperature $T_{\mathrm{g}}$ for many thermoplastics is depressed at lower molecular weights and 35 becomes constant at higher molecular weights [4]. However, in a comprehensive study by Zhang et al. [5], no correlation between $M_{\mathrm{e}}$ and the molecular-weight dependence of $T_{\mathrm{g}}$ was found for polystyrene. Thermodynamic properties such as density $\rho$ and heat capacity (per unit mass) $\hat{c}_{p}$ appear to be relatively insensitive the polymer molecular weight; a weak dependence of these properties on 40 $M$ has been reported in the oligomeric regime $(N \lesssim 10)[6,7]$.

Detailed models for the thermal conductivity of liquids and amorphous solids, while not well developed, often make a connection to sound wave propagation $[8,9,10]$. Based on this, one would expect the dependence of $\lambda$ on molecular weight for polymeric materials to be rather weak. Studies on the 5 molecular weight dependence of thermal conductivity for polymers are, however, relatively small in number. The most well known experimental data is that reported by Hansen and co-workers [11, 12]. In these studies, a rather 
strong dependence of $\lambda$ on $M$ was reported for several commercial polymers having a broad molecular weight distribution. It is worth noting that few deso tails of the experimental method(s) used were provided and the reported data did not include estimates of the experimental uncertainty. In Figure 1 we present one set of results for polystyrene [11, 12], which includes an earlier data set for low molecular weight (oligomeric) polystyrene [13]. From this figure, we see that that $\lambda$ increases by roughly $75 \%$ with $M$ over the range $10^{3}-10^{5} \mathrm{~g} / \mathrm{mol}$.

55 Similar results were reported for high-density polyethylene; however, changes in the 'effective' molecular weight for some samples was achieved by dilution with oligomers (waxes) [11]. We note that these experimental results [11,12] are well cited and have been discussed in a more recent review article [14].

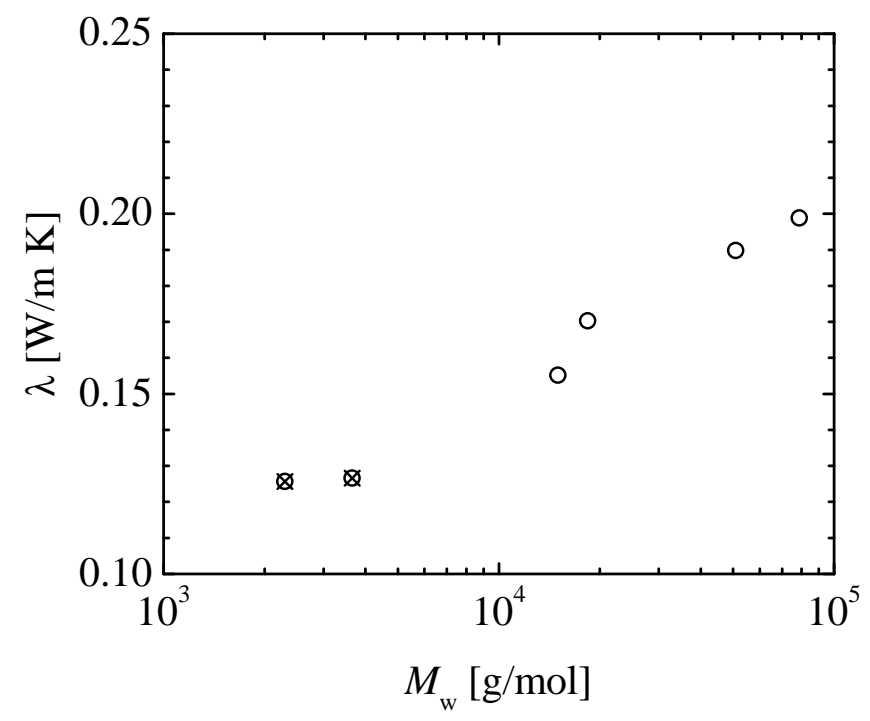

Figure 1: Literature data on the dependence of thermal conductivity $\lambda$ on weight-average molecular weight $M_{\mathrm{w}}$ for polystyrene $40^{\circ} \mathrm{C}$ from [12] $(\bigcirc)$ and [13] $(\otimes)$.

A model proposed by Hansen and Ho [11] that predicts $\lambda \sim \sqrt{N}$ was found to be in good agreement with their experimental results. According to this model, the slope of $\lambda$ vs. $\sqrt{N}$ indicates the relative importance of intra-chain energy transfer compared to inter-chain energy transfer. When intra-chain and interchain energy transfer efficiencies are comparable, $\lambda$ is independent of $\sqrt{N}$ and therefore polymer molecular weight. Hence, these authors claim the observed ${ }_{65}$ dependence of $\lambda$ on $M$ indicates intra-chain energy transfer is more efficient than inter-chain energy transfer. This hypothesis has been the basis for a molecular model for anisotropic thermal conduction in oriented polymeric materials [15]. There appears (to our knowledge) to be only one other experimental data set in the literature where the effect of polymer molecular weight on thermal conduc70 tivity has been examined. Bates reported a weaker dependence of $\lambda$ on molecular 
weight for a series of commercial silicones: tri-methyl end-blocked polydimethyl siloxanes (PDMS) [16], which are reproduced in Figure 2. However, the absence of uncertainties in the measurements makes it difficult to assess whether or not a dependence on molecular weight is present. For completeness, we mention the 75 data set from Morikawa et al. [17] who reported thermal diffusivity $\chi=\lambda / \rho \hat{c}_{p}$ for several polystyrene samples with relatively narrow molecular weight distribution. These data will be discussed later in this paper.

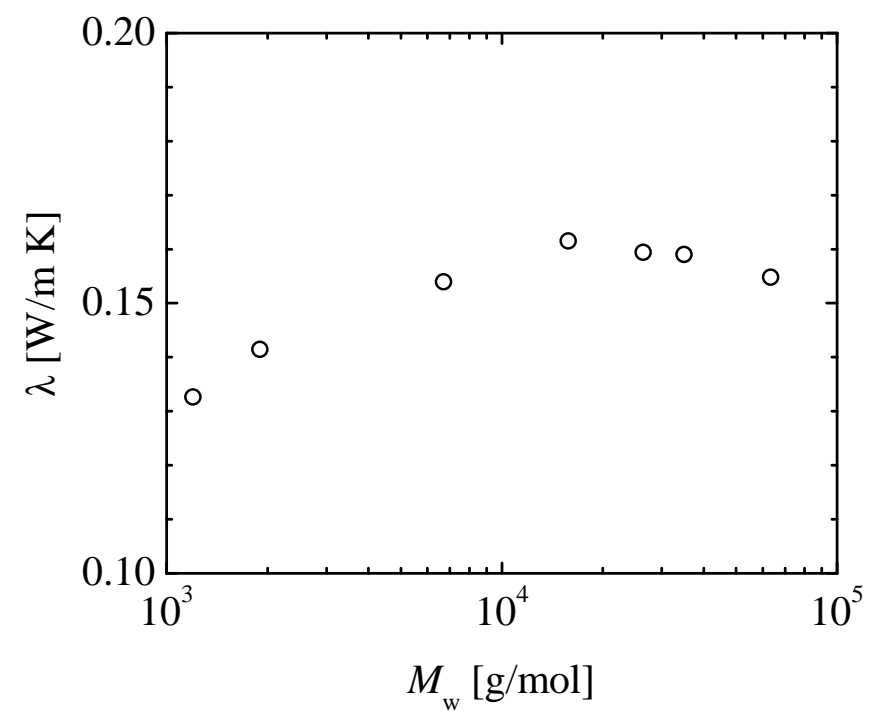

Figure 2: Literature data on the dependence of thermal conductivity $\lambda$ on weight-average molecular weight $M_{\mathrm{w}}$ for polydimethyl siloxane at $50^{\circ} \mathrm{C}$ from [16].

Several molecular dynamics studies on polyethylene melts have found that $\lambda$ increases with $M$ with a similar dependence to that found on the experimental results by Hansen et al. [11]. However, the increase in $\lambda$ is limited to low molecular weights $[18,19,20]$, and all the mentioned simulation studies showed deviations from the proposed $N^{1 / 2}$ model, with a plateau in $\lambda$ over the critical molecular weight or chain length limit $N_{\mathrm{c}}=2 N_{\mathrm{e}}$. Unfortunately, the range of molecular weights covered by these simulations did not correspond to those 85 investigated experimentally [11] due to the computational cost required to equilibrate large molecular weight polymeric melts. A more recent study covered a region overlapping the molecular weight range for both previous simulation $[18,19,20]$ and experimental [11] studies. This study showed three regions for the dependence of thermal conductivity with molecular weight [21]. In a first re90 gion $\left(N<N_{\mathrm{e}}\right), \lambda$ increases roughly as $N^{1 / 2}$; in a second region $\left(N_{\mathrm{e}}<N<N_{\mathrm{c}}\right)$, the rate of increase in thermal conductivity with molecular weight decreases. Finally, above the critical entanglement chain length $\left(N>N_{\mathrm{c}}\right)$ the thermal conductivity remains constant. Note that the constant thermal conductivity found 
in all simulation studies for $N \geq N_{\mathrm{c}}$ is in direct contradiction with Hansen et al. [11] results. These simulation results show good qualitative agreement with a recent model proposed by $\mathrm{Xi}$ et al. [22] with a general expression for the thermal conductivity of amorphous materials ranging from liquids to polymer glasses (i.e., for amorphous solids $\lambda$ is found to be dependent on $M$ and for polymer glasses it is found to be independent). To describe the thermal con-

100 ductivity of polymers, this model makes use of a Thermal Resistance Network that highlights the importance of the distance between entanglements in the path followed by energy carriers along the chains [22, 23]. This model has successfully reproduced the pressure and temperature dependence of $\lambda$ in polymer solids and melts and qualitatively described anisotropy in oriented polymers.

From the discussion above, it appears that the dependence of thermal conductivity on polymer molecular weight has yet to be established. In this study we present a comparison of experiments and molecular dynamic simulations in an attempt to settle the question. We report measurements of thermal conductivity over a wide range of molecular weights for polystyrene (PS) and polyisobutylene (PIB) samples having a narrow molecular weight distribution. We chose these polymers because samples with relatively narrow molecular distribution are readily available for both; results for PS have been previously reported. In addition, PS is one of the few chemistries for which well equilibrated systems used for molecular dynamics simulations can be produced for the wide

${ }_{115}$ range of molecular weights of interest in this study. Thermal conductivity $\lambda$ was determined using a combination of experiments where the density $\rho$, heat capacity $\hat{c}_{p}$ and thermal diffusivity $\chi$ were measured and used in the relation $\lambda=\rho \hat{c}_{p} \chi$. In simulations, we focus on PS at room temperature over a range of molecular weights both below and above $M_{\mathrm{e}}$. In the following sections, we discuss the experimental techniques and simulation methods used. These are followed by a presentation of our results, where we examine the (in)dependence of the thermophysical properties on polymer molecular weight. Finally, we draw conclusions on our results and their implications.

\section{Experimental}

\subsection{Materials and Sample Preparation}

As the focus of this study is on the molecular weight dependence of thermal conductivity, we have chosen to work with samples having a relatively narrow molecular weight distribution. The weight-average molecular weight $M_{\mathrm{w}}$ and polydispersity $M_{\mathrm{w}} / M_{\mathrm{n}}\left(M_{\mathrm{n}}\right.$ number-average molecular weight) values reported in this study are those provided by the suppliers. Most of the PS $\left(M_{0}=104.2 \mathrm{~g} / \mathrm{mol}\right)$ samples were obtained from Polymer Source $\left(M_{\mathrm{w}}=\right.$ $1404,3552,10900,53550,106000,442000 \mathrm{~g} / \mathrm{mol})$; the remaining samples were obtained from Pressure Chemical $\left(M_{\mathrm{w}}=25000 \mathrm{~g} / \mathrm{mol}\right)$, from G. Kisker GbR $\left(M_{\mathrm{w}}=187000 \mathrm{~g} / \mathrm{mol}\right)$, and from Scientific Polymer Products Inc. $\left(M_{\mathrm{w}}=\right.$

$135260000 \mathrm{~g} / \mathrm{mol})$. All but one of the PIB $\left(M_{0}=56.1 \mathrm{~g} / \mathrm{mol}\right)$ samples were obtained from Polymer Source $\left(M_{\mathrm{w}}=1610,3510,7875,34770,81000,130700,449100 \mathrm{~g} / \mathrm{mol}\right)$; 
and one was obtained from BASF (Oppanol B $15 \mathrm{SFN}, M_{\mathrm{w}}=75000 \mathrm{~g} / \mathrm{mol}$ ). A summary of the samples and their molecular weight distribution data is provided in Table 1 . All of the samples have $N>10$. Based on literature values [24] for the entanglement molecular weight $\left(M_{\mathrm{e}}=16,600 \mathrm{~g} / \mathrm{mol}\right.$ for PS and $M_{\mathrm{e}}=6900 \mathrm{~g} / \mathrm{mol}$ for PIB), several of the samples can be classified as unentangled polymer: $M_{\mathrm{w}}<M_{\mathrm{e}}$. Note that all but one of the samples for the PS and PIB series have a relatively narrow molecular weight distribution.

Table 1: Molecular weight and polydispersity data for polystyrene (PS) and polyisobutylene (PIB) samples.

\begin{tabular}{cccccc}
\hline \hline name & $M_{\mathrm{w}}[\mathrm{g} / \mathrm{mol}]$ & $M_{\mathrm{w}} / M_{\mathrm{n}}$ & name & $M_{\mathrm{w}}[\mathrm{g} / \mathrm{mol}]$ & $M_{\mathrm{w}} / M_{\mathrm{n}}$ \\
\hline PS1.4k & 1,404 & 1.08 & PIB1.6k & 1,610 & 1.15 \\
PS3.6k & 3,552 & 1.11 & PIB3.5k & 3,510 & 1.30 \\
PS11k & 10,900 & 1.09 & PIB7.9k & 7,875 & 1.05 \\
PS25k & 25,000 & 1.06 & PIB35k & 34,770 & 1.14 \\
PS54k & 53,550 & 1.05 & PIB75k & 75,000 & 3.4 \\
PS106k & 106,000 & 1.06 & PIB81k & 81,000 & 1.18 \\
PS187k & 187,000 & 1.02 & PIB131k & 130,700 & 1.24 \\
PS260k & 260,000 & 2.5 & PIB499k & 499,100 & 1.14 \\
PS442k & 442,000 & 1.05 & & & \\
\hline
\end{tabular}

The optical method (described below) used to determine the thermal diffusivity requires dust-free, transparent samples that absorb light at the wavelength of $514.5 \mathrm{~nm}$. To produce such samples, the dye 1,4-dihydroxyanthraquinone (quinizarin, 96\%, Aldrich Chemical Company, Inc.) was incorporated at a small mass fraction $\left(\sim 10^{-4}\right)$. To achieve this, the polymer and dye were dissolved in a solvent: ethyl acetate for PS and cyclohexane for PIB at roughly $10 \%$ polymer by mass. The solution was transferred to a mold having a removable glass bottom and a majority of the solvent was allowed to evaporate at room temperature. The resulting films were then placed in a vacuum oven at $50^{\circ} \mathrm{C}$ and dried until the mass of the film was effectively constant, which typically required $2-3$ months. This procedure yielded samples with an absorption co155 efficient $K \leq 20 \mathrm{~cm}^{-1}$ at a wavelength of $514.5 \mathrm{~nm}$. The dyed polymer samples were molded into disks having a diameter $\sim 13 \mathrm{~mm}$ and thickness $\sim 1 \mathrm{~mm}$, which were suitable for the optical method and for the measurement of density. DSC measurements of the heat capacity were made on the as-received polymers after drying in a vacuum oven at $50^{\circ} \mathrm{C}$ for at least $24 \mathrm{hrs}$.

\subsection{Forced Rayleigh Scattering Technique}

Forced Rayleigh Scattering (FRS) is a non-invasive optical technique [25] that can be used to measure thermal diffusivity $\chi$. Over the past 20 years, we have developed and implemented an FRS setup [26] that allows for measurement of the time-dependent components of the thermal diffusivity tensor $\chi=\boldsymbol{\lambda} / \rho \hat{c}_{p}$, where $\boldsymbol{\lambda}$ is the thermal conductivity tensor, in deformed polymeric materials. 
This setup has been used to investigate deformation-induced anisotropic thermal conduction in polymer melts subjected to step and constant rate shear flows $[27,28,29,30,31]$. We have also used this setup to measure components of $\chi$ in several cross-linked elastomers subjected to uniaxial elongation [32, 33, 34, 35], and in polymers quenched after uniaxial elongation in the melt state [36, 37, 38]. Several of the samples listed in Table 1 have been used in our previous work $[27,28,29,31,37]$ on flow-induced anisotropic thermal conduction.

Here we give a brief description of the technique, which is described in detail elsewhere [26]. An optical grating is formed by the intersection of two beams from a coherent $\left(\mathrm{Ar}^{+}\right)$laser within a sample containing a dye that absorbs a small fraction of the energy of the electromagnetic wave. Interference causes the intensity within the intersection volume of the beams, where the sample is located, to be modulated sinusoidally with the period $\Lambda \simeq \lambda^{\mathrm{Ar}^{+}} / \theta$, where $\lambda^{\mathrm{Ar}^{+}}=514.5 \mathrm{~nm}$ is the wavelength of the writing laser and $\theta \ll 1$ is the angle at which the beams cross. The absorbed energy produces a sinusoidal temperature field with a modulation amplitude $\delta T \sim 0.01 \mathrm{~K}$. The temperature modulation leads to a modulation of refractive index $n$, which is detected using a low-power, HeNe reading laser $\left(\lambda^{\mathrm{HeNe}}=632.8 \mathrm{~nm}\right)$ that passes through the sample at the Bragg angle. The intensity of the first-order diffracted beam is measured by a photo-detector.

Since the grating period $\Lambda$ is much smaller than the spot size of the writing laser, the dynamics of the grating temperature field can be decoupled from the bulk temperature in the sample. Following a pulse of the writing laser, $\delta T$ is assumed to have an exponential time dependence: $\delta T \propto e^{-t / \tau_{\mathrm{g}}}$ with grating relaxation time $\tau_{\mathrm{g}}=\frac{\Lambda^{2}}{4 \pi^{2} \chi}$. A fit to the decaying part of the signal is used to obtain a value for $\tau_{\mathrm{g}}$ [26]. Because $\Lambda$ is on the order of tens of microns, the duration of a single experiment is only several milliseconds. Typically, 100 experiments were used to determine an average value of $\tau_{\mathrm{g}}$. FRS experiments were performed at room temperature $\left(\sim 22.5^{\circ} \mathrm{C}\right)$.

195 A check of the validity of assumptions in the physical model used to analyze FRS experiments (i.e., plane grating, Fourier's law, and purely diffusive transport) was be made by varying the grating period $\Lambda$ [26]. Typical results from such a check are shown in Fig. 3 where the expected quadratic dependence of $\tau_{\mathrm{g}}$ on $\Lambda$ is observed. All values of the thermal diffusivity $\chi$ reported in this study were obtained in the manner from data as shown in Fig. 3.

\subsection{Differential Scanning Calorimetry}

Differential scanning calorimetry (DSC) experiments were conducted using either a DSC822 ${ }^{\mathrm{e}}$ (Mettler Toledo) or a DSC 8000 (PerkinElmer) using $\mathrm{N}_{2}$ flow rates of $50 \mathrm{~cm}^{3} / \mathrm{min}$ and $20 \mathrm{~cm}^{3} / \mathrm{min}$, respectively. These experiments were 205 conducted and evaluated according to ASTM E1269 - 11 (2018). The thermal history of samples was erased by heating to $140^{\circ} \mathrm{C}$ (PS) or $100^{\circ} \mathrm{C}$ (PIB) at a heating rate of $20^{\circ} \mathrm{C} / \mathrm{min}$, holding at that temperature for $30 \mathrm{~min}$, and then cooling to room temperature at $40^{\circ} \mathrm{C} / \mathrm{min}$. Baseline, sapphire, and samples were measured under the same conditions described in the following. Samples 


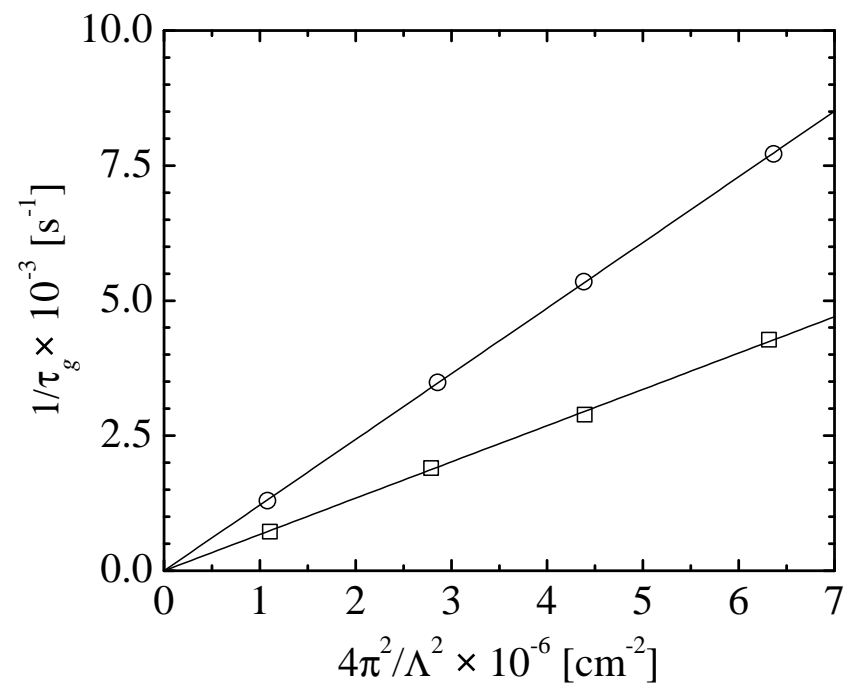

Figure 3: Representative examples of FRS determination of $\chi$ using the relationship $\tau_{\mathrm{g}}=\frac{\Lambda^{2}}{4 \pi^{2} \chi}$ for a polystyrene $(\square)$ and a polyisobutylene $(\bigcirc)$ samples. Each symbol represents an average value derived from 100 individual waveform fits. Uncertainties in both $\tau_{g}$ and $\Lambda$ are smaller than the symbols. The slopes of the lines correspond to the thermal diffusivity $\chi$.

were equilibrated at $-20^{\circ} \mathrm{C}$ for $10 \mathrm{~min}$, heated to $150^{\circ} \mathrm{C}(\mathrm{PS})$ or $100^{\circ} \mathrm{C}(\mathrm{PIB})$ at $10^{\circ} \mathrm{C} / \mathrm{min}$, equilibrated at the respective temperature for $10 \mathrm{~min}$, and cooled to room temperature at $40^{\circ} \mathrm{C} / \mathrm{min}$. No change in sample mass was observed after the measurements. The heat capacity $\hat{c}_{p}$ of the polymer samples was calculated using an approach called the sapphire method, which is also know as ratio or three-curve method [39]. From a propagation of error analysis, we estimate an uncertainty of $6 \%$ for the DSC measurements.

Measured heat capacity $\hat{c}_{p}$ versus molecular weight $M_{\mathrm{w}}$ of PS and PIB at $20^{\circ} \mathrm{C}$ are shown in Figure 4 . From these data it appears that heat capacity is not a function of molecular weight. This is consistent with several studies where a 220 small increase in $\hat{c}_{p}$ was observed for oligomeric $(N<10)$ PS [6, 40]. The average measured $\hat{c}_{p}=1.212 \pm 0.033 \mathrm{~kJ} / \mathrm{kgK}$ for PS and $\hat{c}_{p}=1.900 \pm 0.064 \mathrm{~kJ} / \mathrm{kgK}$ for $\mathrm{PIB}$ are in good agreement with literature values for PS $\left(\hat{c}_{p}=1.192 \mathrm{~kJ} / \mathrm{kgK}\right)$ and PIB $\left(\hat{c}_{p}=1.931 \mathrm{~kJ} / \mathrm{kgK}\right)$ from Gaur et al. [41, 42].

DSC measurements were also used to determine the glass transition temperature $T_{\mathrm{g}}$ of PS as determined by the midpoint value of the endothermic shift. These results are shown in Figure 5 along with DSC results by Blanchard et al. [4] and Zhang et al. [5], all of which are in good agreement. Results from a round robin test based on DSC measurements indicate the value $T_{\mathrm{g}}=107 \pm 2{ }^{\circ} \mathrm{C}$ [43]. In Figure 5, we have also included the estimated $T_{\mathrm{g}}$ from our simulations on polystyrene (see discussion in the Simulation Methods section below). 


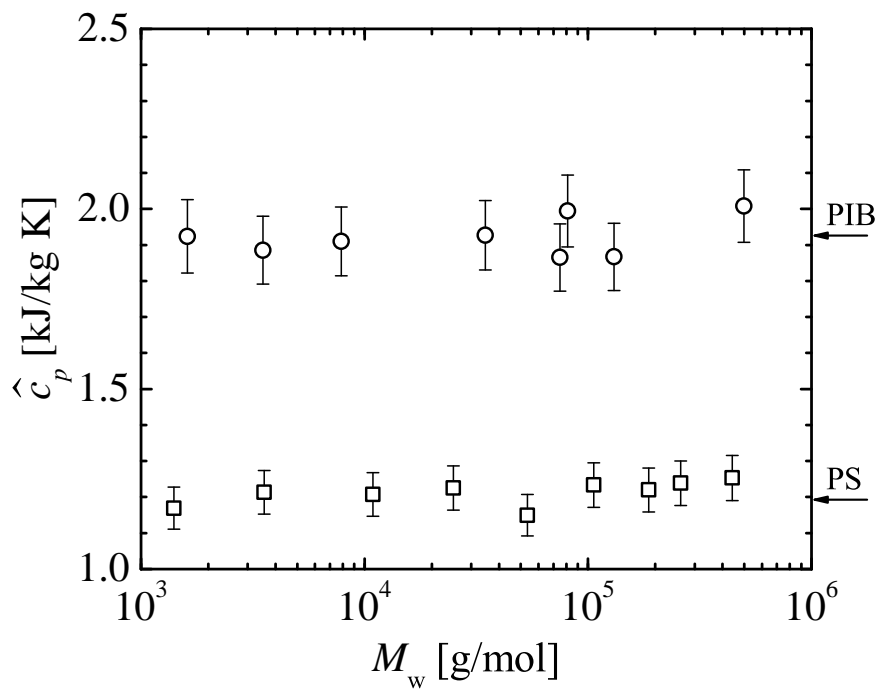

Figure 4: Heat capacity $\hat{c}_{p}$ versus molecular weight $M_{\mathrm{w}}$ for PS $(\square)$ and PIB $(\bigcirc)$ at $20^{\circ} \mathrm{C}$. Arrows represent literature values from Gaur et al. [41, 42].

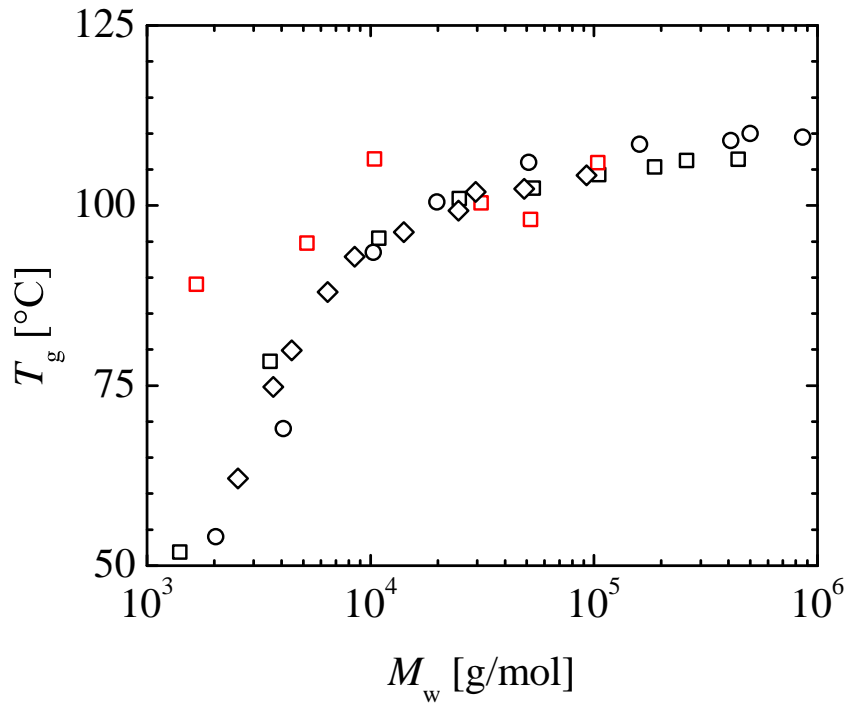

Figure 5: Glass transition temperature $T_{\mathrm{g}}$ vs. the molecular weight $M_{\mathrm{w}}$ of PS as determined by DSC $(\square)$ and by MD simulations $(\square)$. Also shown are $T_{\mathrm{g}}$ measured by DSC from Blanchard et al. [4] $(\bigcirc)$ and from Zhang et al. [5] $(\diamond)$. 


\subsection{Densimetry}

The mass density $\rho$ of the polymer samples was determined using compression molded PS disks and degassed PIB melts. These measurements were made at $20^{\circ} \mathrm{C}$ using a Gay-Lussac pycnometer $\left(V=2 \pm 0.05 \mathrm{~cm}^{3}\right.$, Ace Glass Inc.) in combination with a thermo-stated water bath. For PS, distilled water $\left(\rho^{20^{\circ} \mathrm{C}}=0.9982 \mathrm{~g} / \mathrm{cm}^{3}\right)$ served as displacement liquid; PIB was immersed in 2-propanol $\left(\rho^{20^{\circ} \mathrm{C}}=0.7851 \mathrm{~g} / \mathrm{cm}^{3}\right)$ due to its low density [44]. For the lower molecular weight PIB samples, where the tackiness of PIB caused difficulties, water was used to verify the measurements in 2-propanol. The uncertainty of mass density measurements is estimated to be less than one $\%$.

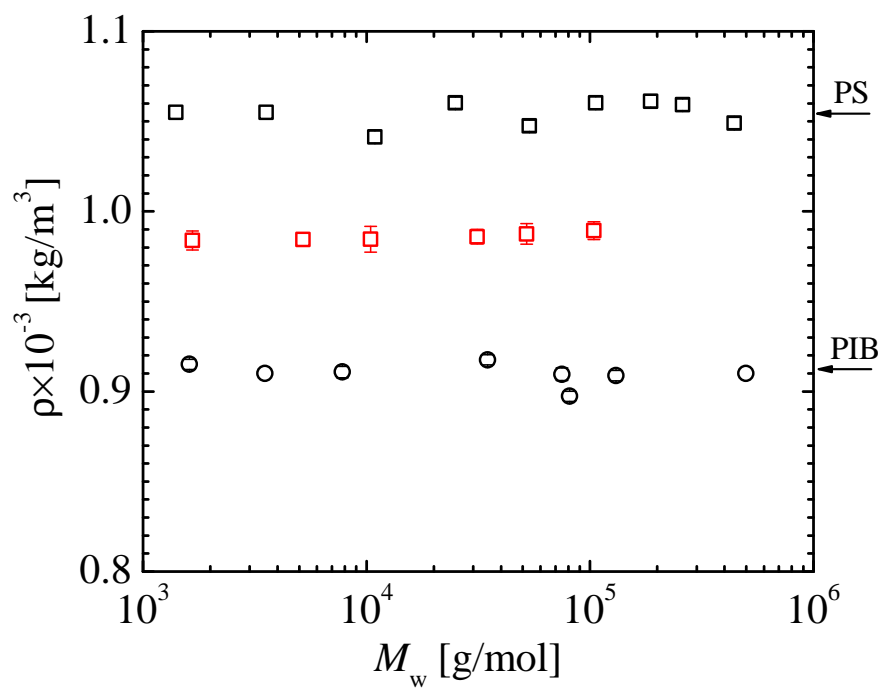

Figure 6: Mass density $\rho$ versus molecular weight $M_{\mathrm{w}}$ for PS ( $\square$ for experiments and $\square$ for simulations) and PIB $(\bigcirc)$ at $20^{\circ} \mathrm{C}$. Arrows represent literature values [7].

Figure 6 shows the measured mass density $\rho$ versus molecular weight for the PS and PIB samples. From this figure, no dependence on molecular weight is displayed for either PS or PIB. It has been reported that, below the glass transition temperature $T_{\mathrm{g}}$, density $\rho$ is independent of $M_{\mathrm{w}}[7,45]$. Results reported by Sangalov et al. for PIB above $T_{\mathrm{g}}$ indicate $\rho=0.83-0.91 \mathrm{~g} / \mathrm{cm}^{3}$ for $M_{\mathrm{w}}<50000 \mathrm{~g} / \mathrm{mol}$ and $\rho=0.91-0.93 \mathrm{~g} / \mathrm{cm}^{3}$ for $M_{\mathrm{w}}=70000-225000 \mathrm{~g} / \mathrm{mol}$ [46]. However, it is difficult to ascribe a clear dependence of $\rho$ on $M_{\mathrm{w}}$ from these results. From the data shown in Figure 6, we find the average for PS of $\rho=1.054 \pm 0.008 \mathrm{~g} / \mathrm{cm}^{3}$ is in good agreement with the literature value ${ }_{250} \rho=1.0549 \pm 0.0003 \mathrm{~g} / \mathrm{cm}^{3}[7]$. For PIB, we find an average value of $\rho=$ $0.912 \pm 0.003 \mathrm{~g} / \mathrm{cm}^{3}$, which is within the range of literature values [46]. In Figure 6 , we have included simulation results for the equilibrium density at 300 $\mathrm{K}$. We find $\rho=0.985 \pm 0.004 \mathrm{~g} / \mathrm{cm}^{3}$ - unaffected by polydispersity as shown by 
Table 2: Characteristics of the simulated polystyrene systems. Note that for mono-disperse systems $M_{\mathrm{w}} / M_{\mathrm{n}}=1, M_{\mathrm{w}}=M$

\begin{tabular}{lccccc}
\hline \hline name & $N$ & $M_{\mathrm{w}}$ & $M_{\mathrm{w}} / M_{\mathrm{n}}$ & Distribution & $N_{\text {chains }}$ \\
\hline m-PS16 & 16 & 1,700 & 1 & monodisperse & 150 \\
m-PS50 & 50 & 5,200 & 1 & monodisperse & 20 \\
m-PS100 & 100 & 10,400 & 1 & monodisperse & 10 \\
m-PS300 & 300 & 31,200 & 1 & monodisperse & 10 \\
m-PS500 & 500 & 52,100 & 1 & monodisperse & 5 \\
m-PS1000 & 1000 & 140,200 & 1 & monodisperse & 3 \\
u-PS50 & 50 & 5,200 & 1.1 & uniform & 60 \\
b-PS50 & 54 & 5,600 & 1.12 & bimodal $(50,150)$ & $54 \& 2$ \\
u-PS100 & 100 & 10,400 & 1.1 & uniform & 30 \\
b-PS100-1 & 115 & 12,000 & 1.1 & bimodal $(100,200)$ & $22 \& 4$ \\
b-PS100-2 & 107 & 11,200 & 1.12 & bimodal $(100,300)$ & $27 \& 1$ \\
\hline
\end{tabular}

Figure S6 in the Supporting Information - which is about 7\% lower than the Carlo (CAMC) simulations at $500 \mathrm{~K}[50,51]$. CAMC uses a combination of moves which allows for large jumps in the configuration space. As a result, the different configurations of the material can be sampled more efficiently than 
temperature. The microscopic heat flux vector can be written as [21]

$$
\boldsymbol{J}=\frac{1}{V} \frac{\mathrm{d}}{\mathrm{d} t} \sum_{i=1}^{N} \varepsilon_{i} \boldsymbol{r}_{i}
$$

where the energy $\varepsilon_{i}$ carried by each particle $(i=1, \ldots N)$ at $\boldsymbol{r}_{i}$ with instantaneous velocity $\boldsymbol{v}_{i}$ is given by:

$$
\varepsilon_{i}=\frac{p_{i}^{2}}{2 m_{i}}+\mathcal{V}_{i}
$$

Equation (4) separates kinetic and potential energy contributions of each particle. The kinetic energy contribution is given by the mass $m_{i}$ and momentum 315

using standard molecular dynamics simulations. Once equilibrium at the coarsecovered via a reverse-mapping procedure that preserves both tacticity and the long-range conformational characteristics of the chains [47]. Although hydrogen been shown to fully capture the structure dynamics as well as the mechanical properties of atactic polystyrene [47, 51, 53]. In order to compare simulation and experimental results, the configurations obtained at $500 \mathrm{~K}$ are cooled using ns) and $25 \mathrm{~K}$ quenching $(2.5 \mathrm{~ns}$ ) steps. For example, after energy minimization reached steady-state by the step's end. The equilibration step is followed by a configurations extracted at the end of each equilibration step are considered at in experiments $\sim 295 \mathrm{~K}$. We note that variations in the thermal conductivity due to temperature changes around the experimental conditions are within the thermal conductivity as a function of temperature for all studied monodisperse melts has been included in Figure S5 in the Supporting information.

The equilibrium thermal conductivity of the well equilibrated configurations is obtained using the equilibrium molecular dynamics Green-Kubo (G-K) ductivity tensor components are given by the autocorrelation function of the microscopic heat flux components $J_{i}$ in the $i$-direction.

$$
\lambda_{\alpha \beta}=\frac{V}{k_{\mathrm{B}} T^{2}} \int_{0}^{\infty} J_{\alpha}(0) J_{\beta}(0) d t,
$$

$\boldsymbol{p}_{i}$ of particle $i$. The potential energy contribution $\mathcal{V}_{i}$ includes contributions by 
pairs (bonded and non-bonded stretching energy), triplets (bond angle bending energy) and quadruplets (dihedral and improper torsion energy). Additional details on the G-K method the interaction potentials included in $\mathcal{V}_{i}$ is given elsewhere [21, 47].

In addition to the thermal conductivity, we have computed the average density at the end of the equilibration steps for all melts for temperature ranging from $275 \mathrm{~K}$ to $500 \mathrm{~K}$. The density of the equilibrated systems as a function of temperature has been included in Figure S4 in the Supporting Information. We find the value of $T_{\mathrm{g}}$ for each molecular weight by computing the intersection 325 of linear fits to the two well-differentiated regimes above and below the transition. The determination of $T_{\mathrm{g}}$ is rate dependent and therefore, comparison of simulation and experimental results should be understood as qualitative [55]. However, we find the value for all the melts with $N>N_{\mathrm{c}}$ to be in close proximity to the experimental consensus $T_{\mathrm{g}} \sim 100^{\circ} \mathrm{C}[56,57]$. We also find qualitative agreement with the experimental results shown in Figure 5: $T_{\mathrm{g}}$ from simulations decreases with decreasing molecular weight.

All the results for the thermal conductivity, density and glass transition temperature of melts correspond to the average of three independent configurations. Equilibration, quenching and thermal conductivity measuring steps were all performed using the LAMMPS MD package [58].

\section{Results and Discussion}

In this section, we present results for the thermal transport properties of PS and PIB samples as a function of molecular weight. As shown in the preceding sections, the thermodynamic properties $\left(\rho\right.$ and $\hat{c}_{p}$ ) required to compute the thermal conductivity $\lambda$ from the measured values of $\chi$ show no dependence on the molecular weight. In addition, we compare the calculated values of $\lambda$ with those obtained from molecular dynamics simulations.

Thermal diffusivity $\chi$ versus molecular weight results are shown in Figure 7 for PS and PIB samples. At high molecular weights, both polymers show 345 a region where $\chi$ is independent of the molecular weight (i.e., all values are within the measurement uncertainty - in agreement with the average value in that region). At lower molecular weights, both polymers show a weak dependence on molecular weight, albeit only two points for PS and three for PIB are in this region. In particular, PS1.4k and PIB1.6k show respectively $10 \%$ and $7 \%$ 350 smaller $\chi$ than the samples with larger molecular weight. These differences are significant since the estimated uncertainty of the FRS measurements of thermal diffusivity is $\sim 1 \%$. We place the transition between both regimes somewhere between $M_{\mathrm{w}} \sim 10,000 \mathrm{~g} / \mathrm{mol}$ and $30,000 \mathrm{~g} / \mathrm{mol}$, which is comparable to $M_{\mathrm{c}}$ for both polymers. Note that PS1.4k and PIB1.6k are also somewhat close to the 355 oligomeric regime with $N \simeq 14$ and $N \simeq 29$ respectively. In Figure 7 , we also included previous results by Morikawa et al. [17] for the thermal diffusivity of PS. Their measurements show absolute values that are roughly $10 \%$ larger than those from the present study, but indicate a similar trend with a $12 \%$ reduction in $\chi$ at low molecular weights. However, the absence of uncertainty in their 


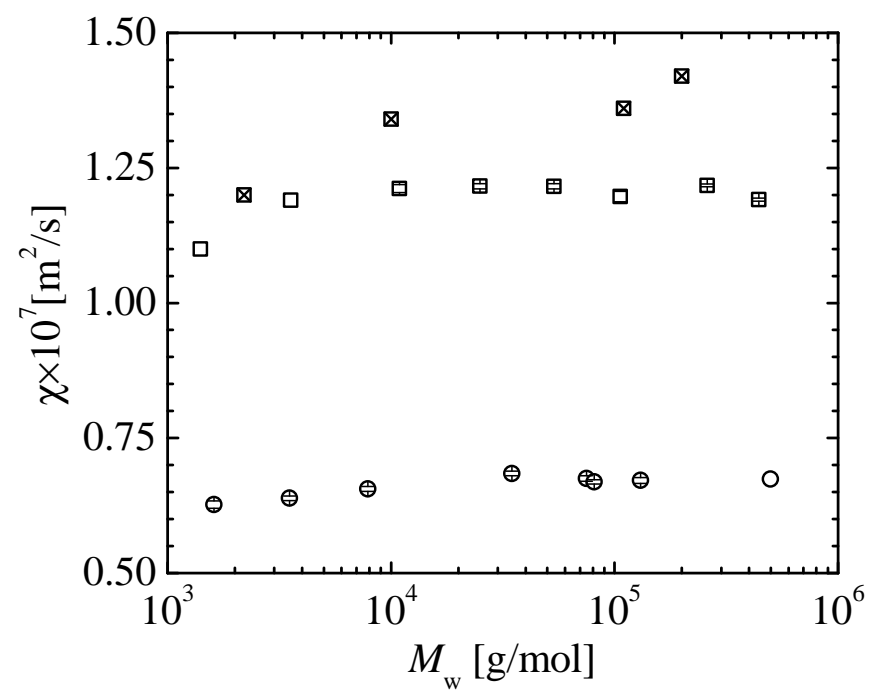

Figure 7: Thermal diffusivity $\chi$ versus molecular weight $M_{\mathrm{w}}$ for PS ( $\square$ ) and PIB (O) at $22.5^{\circ} \mathrm{C}$. Also shown is data from Morikawa et al. for PS ( $\left.\otimes\right)$ at room temperature [17].

Having presented our measurements of mass density $\rho$, heat capacity $\hat{c}_{p}$, and thermal diffusivity $\chi$, we proceed with thermal conductivity calculated according to $\lambda=\rho \hat{c}_{p} \chi$. These results are shown in Figure 8 where $\lambda$ is plotted versus $M_{\mathrm{w}}$ for PS and PIB. The uncertainties in measurements of $\rho, \hat{c}_{p}$, and $\chi$ propagate into the calculated values of $\lambda$ making it more difficult to examine its dependence on molecular weight. In other words, the uncertainty in $\chi$ of $\sim 1 \%$ is much smaller than the uncertainty in $\lambda$ of $\sim 6 \%$. Nevertheless, PS1.4k sample shows a noticeable decrease in $\lambda$ and a slightly decreasing trend can be observed in thermal conductivity of PIB for the three lowest molecular weights.

For thermal conductivity, we average our measurements for $M_{\mathrm{w}} \geq 10000 \mathrm{~g} / \mathrm{mol}$, which is approximately $M_{\mathrm{e}}$ for both polymers, and find the following values: $\lambda=0.155 \pm 0.004 \mathrm{~W} / \mathrm{mK}$ for PS and $\lambda=0.118 \pm 0.004 \mathrm{~W} / \mathrm{mK}$ for PIB. As noted in the introduction, literature values for $\lambda$ show a large variation. However, our 375 results are in good agreement with literature values for PS $\lambda=0.154 \mathrm{~W} / \mathrm{m} \mathrm{K}$ based on data from several sources [59] and for PIB $\lambda=0.13 \mathrm{~W} / \mathrm{m} \mathrm{K}[1,46]$. For convenience, we have listed all of the measured thermophysical properties for PS in Table S1 and for PIB in Table S2 in the Supporting Information.

Thermal conductivity determined from our molecular dynamics simulations are also included in Figure 8 for PS systems having molecular weights ranging from $N=16(M=1,700 \mathrm{~g} / \mathrm{mol})$ to $N=1000(M=104,000 \mathrm{~g} / \mathrm{mol})$. To our knowledge this is the most extensive simulation study of the thermal conduc- 


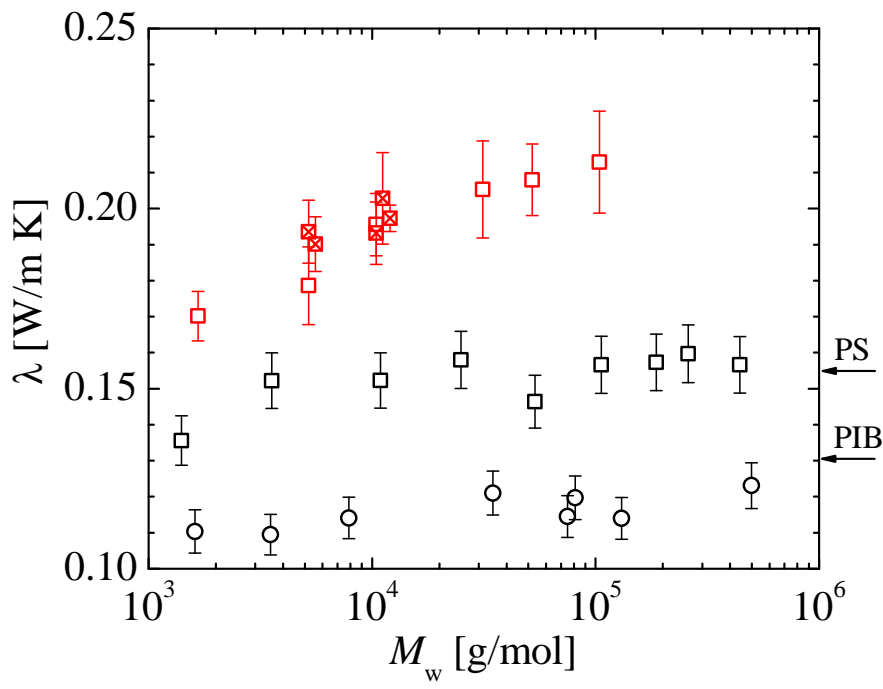

Figure 8: Thermal conductivity $\lambda$ versus molecular weight $M_{\mathrm{w}}$. Experimental results include PS $(\square)$ and PIB $(\bigcirc)$ samples at $20^{\circ} \mathrm{C}$. Simulation results include monodisperse where $M_{\mathrm{w}}=$ $M(\square)$ and polydisperse ( $\otimes)$ PS systems at $300 \mathrm{~K}$. Arrows represent literature values for PS [59] and PIB [1, 46].

tivity of PS above the oligomeric regime and above the entanglement molecular weight. We observe that above $M_{\mathrm{c}}$ the thermal conductivity from simulations reaches an effectively constant value $\lambda=0.209 \pm 0.003$, which is about $30 \%$ larger than the experimental results shown in Figure 8. Simulation results in Figure 8 also show a stronger dependence on molecular weight below the critical molecular weight - thermal conductivity decreases by $18 \%$ for the lowest molecular weight while it only decreases by $10 \%$ in the experimental data. These discrepancies suggest that refinements in the employed force field [47] might be required to fully match experimental results. Nevertheless, both experimental and simulation results show no dependence at high molecular weights and a weak dependence at lower molecular weights.

We studied polydispersity as possible explanation for the differences between experimental and simulation results. A small number of long chains embedded in a collection of low molecular weight chains might shift the thermal conductivity closer to that of an high molecular system. To examine this, we simulated polydisperse melts with PDI $\sim 1.1$, which corresponds to the value observed in most of the experimental samples. Table 2 summarizes the characteristic 400 parameters for the molecular weight distribution of each simulated system in Figure 8, although additional data for systems with different average molecular weights are included in Table S4 in the Supporting Information. To introduce polydispersity, we have considered two approaches: 1) to generate "uniform" (u) distributions around the target average molecular weights and 2) to include 
405

a few chains of high molecular weigh in a monodisperse low molecular weight melt resulting in a "bimodal" (b) distribution that mimics a long tail in the molecular weight distribution. In all the polydisperse systems, we kept the total number of monomers constant so that the system size is not a factor in the simulations. Note that the preparation of low molecular weight polydisperse 410 samples is limited by the size of the system, if one does not want to significantly increase polydispersity. Results for polydisperse samples in Figure 8 indicate polydispersity has no discernable effect on thermal conductivity (also see Figure S3 in the Supporting information), which is consistent with the experimental results in this figure.

\section{Conclusions}

In conclusion, we have performed careful experiments and simulations to study the dependence of thermo-physical properties of amorphous polymers on molecular weight. The satisfactory agreement between experimental and simulation results for amorphous PS should encourage the use of similar approaches to study the macroscopic properties of polymeric materials using molecular dynamics. Contrary to previous studies, our results show the thermal conductivity to be constant in the high molecular weight region with weak dependence of thermal conductivity on molecular weight between the oligomeric and entangled regions (i.e., at low molecular weights). Our results raise questions about the ${ }_{425}$ hypothesis of preferential intra-chain transport of energy in amorphous polymers, which would manifest as a strong dependence of thermal conductivity on molecular weight. This gap in understanding needs to be addressed if we are to explain more complex phenomena such as the anisotropy in thermal conductivity displayed by polymers when subjected to deformation.

\section{Acknowledgements}

The authors acknowledge NSF Grant No. CBET-1336442, and a grant from the EU's Horizon 2020 Research and Innovation Program under the Marie Sklodowska-Curie IF MTCIATTP 750985, for partial support of this study.

\section{References}

[1] Y. Yang, Thermal conductivity, in: J. E. Mark (Ed.), Physical Properties of Polymers, 2nd Edition, Springer, New York, 2007, pp. 155-163.

[2] D. Hands, The thermal transport properties of polymers, Rubber Chem. Tech. 50 (1977) 480-522.

[3] M. Rubinstein, R. H. Colby, Polymer Physics, Oxford University Press, New York, 2003. 
[4] L. Blanchard, J. Hesse, S. L. Malhotra, Effect of molecular weight on glass transition by differential scanning calorimetry, Can. J. Chem. 52 (1974) 3170-3175.

[5] L. Zhang, J. A. Marsiglio, T. Lan, J. M. Torkelson, Dramatic tunability of the glass transition temperature and fragility of low molecular weight polystyrene by initiator fragments located at chain ends, Macromol. 46 (2016) 2387-2398.

[6] B. V. Kokta, J. L. Valade, V. Hornof, K. N. Law, Effect of molecular weight of polystyrene on heat capacity and thermal transitions, Thermochim. Acta $14(1-2)(1976) 71-86$.

[7] T. G. Fox Jr., P. J. Flory, Second-order transition temperatures and related properties of polystyrene. I. Influence of molecular weight, J. Appl. Phys. 21 (6) (1950) 581-591.

[8] D. G. Cahill, S. K. Watson, R. O. Pohl, Lower limit to the thermal conductivity of disordered crystals, Phys. Rev. B 46 (1992) 6131-6140.

[9] R. Orbach, Vibrational transport in disordered systems, PMB 65 (1992) 289-301.

[10] W.-P. Hsieh, M. D. Losego, P. V. Braun, S. Shenogin, P. Keblinski, D. G. Cahill, Testing the minimum thermal conductivity model for amorphous polymers using high pressure, RB 83 (2011) 174205.

[11] D. Hansen, C. C. Ho, Thermal conductivity of high polymers, J. Polym. Sci. Part A 3 (1965) 659-670.

[12] D. Hansen, R. C. Kantaya, C. C. Ho, Thermal conductivity of high polymers - the influence of molecular weight, Poly. Eng. Sci. 6 (3) (1966) 260262.

[13] K. Ueberreiter, E. O. Laupenmuhlen, Spezifische Wrme, spezifisches Volumen, Temperatur- und Wrmeleitfhigkeit von Hochpolymeren, Z. Naturforsch. A 8A (1953) 664-673.

[14] A. Henry, Thermal transport in polymers, Annu. Rev. Heat Trans. 17 (2014) 485-520.

[15] B. H. A. A. van den Brule, A network theory for the thermal conductivity of an amorphous polymeric material, Rheol. Acta 28 (1989) 257-266.

[16] O. K. Bates, Thermal conductivity of liquid silicones, Ind. Eng. Chem. 41 (9) (1949) 1966-1968.

[17] J. Morikawa, J. Tan, T. Hashimoto, Study of change in thermal diffusivity of amorphous polymers during glass transition, Polymer 36 (23) (1995) 4439-4443. 
[18] J. Zhao, J.-W. Jiang, N. Wei, Y. Zhang, T. Rabczuk, Thermal conductivity dependence on chain length in amorphous polymers, J. Appl. Phys. 113 (2013) 184304.

[19] T. Zhang, T. Luo, Role of chain morphology and stiffness in thermal conductivity of amorphous polymers, J. Phys. Chem. B 120 (2016) 803-812.

[20] J. Wei, T. Luo, Chain length effect on thermal transport in amorphous polymers and a structure thermal conductivity relation, Phys. Chem. Chem. Phys. 21 (2019) 15523-15530.

[21] D. Nieto Simavilla, A. P. Sgouros, G. G. Vogiatzis, C. Tzoumanekas, V. G. W. M. H. Verbeeten, D. N. Theodorou, Molecular dynamics test of the stress-thermal rule in polyethylene and polystyrene entangled melts, Macromol. 53 (2020) 789-802.

[22] Q. Xi, J. Zhong, J. He, X. Xu, Y. Wang, J. Liu, B. Li, T. Nakayama, A ubiquitous thermal conductivity formula for liquids, polymer glass, and amorphous solids, Chinese Physics Letters 37 (2020) 104401. doi:10.1088/ 0256-307X/37/10/104401.

[23] J. Zhou, Q. Xi, J. He, X. Xu, T. Nakayama, Y. Wang, J. Liu, Thermal resistance network model for heat conduction of amorphous polymers, Phys. Rev. Materials 4 (2020) 015601. doi:10.1103/PhysRevMaterials . 4.015601.

[24] L. Fetters, D. Lohse, R. Colby, Chain dimensions and entanglement spacings, in: J. E. Mark (Ed.), Physical Properties of Polymers, 2nd Edition, Springer, New York, 2007, pp. 447-454.

[25] H. J. Eichler, P. Gunter, D. W. Pohl, Laser-Induced Dynamic Gratings, Springer-Verlag, Berlin, 1986.

[26] D. C. Venerus, J. D. Schieber, H. Iddir, J. D. Guzmán, A. W. Broerman, Measurement of thermal diffusivity in polymer melts using forced rayleigh light scattering, J. Polym. Sci., Polym. Phys. Ed. 37 (1999) 1069-1078.

[27] D. C. Venerus, J. D. Schieber, H. Iddir, J. D. Guzmán, A. W. Broerman, Relaxation of anisotropic thermal diffusivity in a polymer melt following step shear strain, Phys. Rev. Lett. 82 (1999) 366-369.

[28] D. C. Venerus, J. D. Schieber, H. Iddir, J. D. Guzmán, A. W. Broerman, Anisotropic thermal diffusivity measurements in deforming polymers and the stress-thermal rule, Int. J. of Thermophys. 22 (2001) 1215-1225.

[29] D. C. Venerus, J. D. Schieber, V. Balasubramanian, K. Bush, S. Smoukov, Anisotropic thermal conduction in a polymer liquid subjected to shear flow, Phys. Rev. Lett. 93 (2004) 098301. 
[30] J. D. Schieber, D. C. Venerus, K. Bush, V. Balasubramanian, S. Smoukov, Measurement of anisotropic energy transport in flowing polymers by using a holographic technique, Proc. Natl. Acad. Sci. U.S.A. 36 (2004) 1314213146 .

[31] V. Balasubramanian, K. Bush, S. Smoukov, D. C. Venerus, J. D. Schieber, Measurements of flow-induced anisotropic thermal conduction in a polyisobutylene melt following step shear flow, Macromol. 38 (2005) 6210-6215.

[32] A. W. Broerman, D. C. Venerus, J. D. Schieber, Evidence for the stressthermal rule in an elastomer subjected to simple elongation, J. Chem. Phys. 111 (1999) 6965-6969.

[33] D. C. Venerus, D. N. Kolev, Anisotropic thermal conductivity in crosslinked polybutadienes subjected to uniaxial elongation, Macromol. 42 (2009) 2594-2598.

[34] D. Nieto Simavilla, J. D. Schieber, D. C. Venerus, Anisotropic thermal transport in a cross-linked polyisoprene rubber subjected to uniaxial elongation, J. Polym. Sci. Part B 50 (2012) 1638-1644.

[35] D. C. Venerus, D. N. Simavilla, J. D. Schieber, Thermal transport in crosslinked elastomers subjected to elongational deformations, Rubber Chemistry and Technology 92 (4) (2019) 639-652. doi:10.5254/rct.19.80382.

[36] J. D. Schieber, D. C. Venerus, S. Gupta, Molecular origins of anisotropy in thermal conductivity in deformed polymer melts, Soft Matter 8 (2012) 11781-11785.

[37] S. Gupta, J. D. Schieber, D. C. Venerus, Anisotropic thermal conduction in polymer melts in uniaxial elongation flows, J. Rheol. 57 (2013) 427-439.

[38] D. C. Venerus, A. Agarwal, Thermal conductivity of poly(L-lactic acid) subjected to elongational deformations, J. Polym. Sci. Part B 57 (2018) $547-553$.

[39] M. J. O'Neill, Measurement of specific heat functions by differential scanning calorimetry, Anal. Chem. 38 (10) (1966) 1331-1336.

[40] S. S. Chang, Thermodynamic properties and glass transition of polystyrene, J. Polym. Sci. Polym. Symp. 71 (1) (1984) 59-76.

[41] U. Gaur, B. Wunderlich, Heat capacity and other thermodynamic properties of linear macromolecules. V. Polystyrene, J. Phys. Chem. Ref. Data 11 (2) (1982) 313-325.

[42] U. Gaur, B. B. Wunderlich, B. Wunderlich, Heat capacity and other thermodynamic properties of linear macromolecules. VII. Other carbon backbone polymers, J. Phys. Chem. Ref. Data 12 (1) (1983) 29-63. 
[43] J. Rieger, The glass transition temperature of polystyrene, J. Therm. Anal. Calorim. 46 (3-4) (1996) 965-972.

[44] U. Hübschmann, E. Links, Tabellen zur Chemie in Ausbildung und Beruf, Handwerk und Technik GmbH, Hamburg, 1998.

[45] E. J. Merz, L. E. Nielsen, R. Buchdahl, Influence of molecular weight on the properties of polystyrene, Ind. Eng. Chem. 43 (6) (1951) 1396-1401.

[46] Y. A. Sangalov, K. S. Minsker, G. E. Zaikov, Polymers derived from Isobutylene: Synthesis, properties, application, VSP, Utrecht, 2001.

[47] G. G. Vogiatzis, D. N. Theodorou, Local segmental dynamics and stresses in polystyrene C60 mixtures, Macromol. 47 (2014) 387-404.

[48] G. Zhang, A. Chazirakis, V. A. Harmandaris, T. Stuehn, K. C. Daoulas, K. Kremer, Hierarchical modelling of polystyrene melts: from soft blobs to atomistic resolution, Soft Matter 15 (2019) 289-302.

[49] I. G. Mathioudakis, G. G. Vogiatzis, C. Tzoumanekas, D. N. Theodorou, Molecular modeling and simulation of polymer nanocomposites at multiple length scales, 2015 IEEE 15th International Conference on Nanotechnology (IEEE-NANO) (2015) 1448-1452.

[50] V. G. Mavrantzas, D. N. Theodorou, Atomistic simulation of polymer melt elasticity: Calculation of the free energy of an oriented polymer melt, Macromol. 31 (1998) 6310-6332.

[51] T. Spyriouni, C. Tzoumanekas, D. Theodorou, F. Müller-Plathe, G. Milano, Coarse-grained and reverse-mapped united-atom simulations of long-chain atactic polystyrene melts: Structure, thermodynamic properties, chain conformation, and entanglements, Macromol. 40 (2007) 3876-3885.

[52] A. V. Lyulin, M. A. J. Michels, Molecular dynamics simulation of bulk atactic polystyrene in the vicinity of Tg, Macromol. 35 (2002) 1463-1472.

[53] N. Lempesis, G. G. Vogiatzis, G. C. Boulougouris, L. C. van Breemen, M. Hütter, D. N. Theodorou, Tracking a glassy polymer on its energy landscape in the course of elastic deformation, Mol. Phys. 111 (2013) 34303441 .

[54] D. J. Evans, G. Morriss, Statistical Mechanics of Nonequilibrium Liquids, 2nd Edition, Cambridge University Press, Cambridge, 2008.

[55] S. V. Kallivokas, A. P. Sgouros, D. N. Theodorou, Molecular dynamics simulations of epon-862/detda epoxy networks: structure, topology, elastic constants, and local dynamics, Soft Matter 15 (2019) 721-733. doi:10. 1039/C8SM02071J. 
[56] T. G. Fox, P. J. Flory, Secondorder transition temperatures and related properties of polystyrene. i. influence of molecular weight, Journal of Applied Physics 21 (6) (1950) 581-591. doi:10.1063/1.1699711.

[57] J. Rieger, The glass transition temperature of polystyrene, Journal of thermal analysis 46 (3) (1996) 965-972. doi:10.1007/BF01983614.

[58] S. Plimpton, Fast parallel algorithms for short-range molecular dynamics, J. Comput. Phys. 117 (1995) 1-19.

595 [59] L. C. K. Carwile, H. J. Hoge, Thermal conductivity of polystyrene: Selected values, Tech. rep., Army Pioneering Research Laboratory, Natick, MA (1966). 